\title{
N94-20503
}

\section{COPLANAR WAVEGUIDE RADIAL LINE STUB}

R. N. Simons and S. R. Taub

Indexing terms: Waveguldes, Resonators, Silicon

A coplanar waveguide radial line stub resonator is experimentally characterised with respect to stub radius, sectora angle, substrate thickness and relative dielectric constant. A simple closed-form design equation, which predicts the resonance radius of the stub, is presented.

Introduction: Coplanar waveguide (CPW) technology is emerging as a viable alternative to stripline and microstrip line for monolithic microwave integrated circuits [1]. There are several applications in which a CPW radial line stub resonator is necessary; these include: bias line filters requiring a point of virtual RF ground, mixer and frequency multiplier circuits that require a reactance to terminate the diodes and the harmonics, respectively. In addition, a grounded CPW

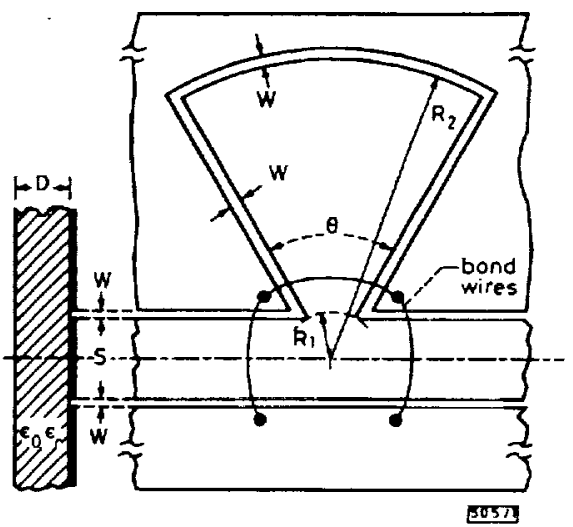

Fig. 1 Schematic diagram of CPW radial line stub

(GCPW) radial line stub resonator has application in oscillator circuits for active antennas [2]. In general, the advantages of a radial line stub over a straight $50 \Omega$ stub are the smaller resonance length, wider bandwidth and smaller discontinuity reactance at the junction with the main line [3].

This Letter presents, for the first time, CPW and GCPW radial line stub resonators, characterised by measuring the resonance frequency. The resonance frequency is measured for stubs with different radii, sectoral angles, substrate thicknesses and substrate relative dielectric constants. A closed-form design equation, which predicts the resonance radius of the
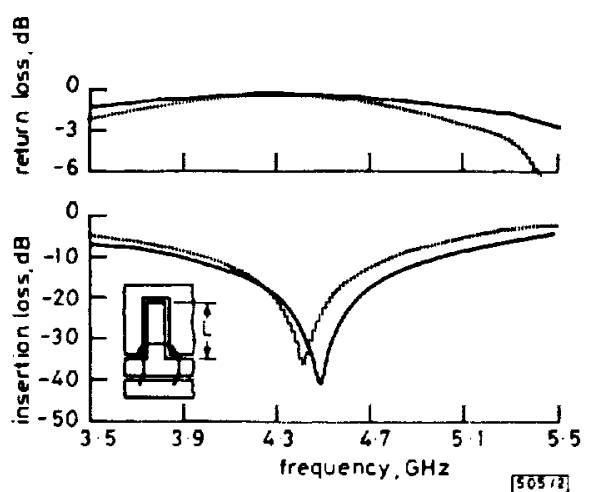

Fig. 2 Measured return loss and insertion loss of $C P W$ radial line stub and $C P W$ straight stub

$S=0.33 \mathrm{~mm}, W=0.234 \mathrm{~mm}, D=0.635 \mathrm{~mm}, \varepsilon_{r}=10.5, R_{1}=$ $0.191 \mathrm{~mm}, R_{2}=5.08 \mathrm{~mm}, \theta=60^{\circ}, L=7 \mathrm{~mm}$

radial line stub

-..... straight stub

(C) Reprinted, with petmission, from IEE Electronics Letters, vol. 29, no. 4, 18th February 1993, pp. 412-413. stub, for a given set of parameters, is also presented.

Design and fabrication: Fig. 1 shows a schematic diagram of the $\mathrm{CPW}$ radial line stub resonator. The inner and outer radius of the stub are denoted as $R_{1}$ and $R_{2}$, respectively. The sectoral angle is denoted as $\theta$. The $C P W$ stubs are fabricated on Duroid (relative dielectric constants $\varepsilon_{r}=6$ and 10.5) and on high resistivity silicon $\left(\varepsilon_{r}=11 \cdot 7, \rho=5000-10000 \Omega \mathrm{cm}\right)$ substrates. The GCPW stubs are labricated on Duroid with $\varepsilon_{r}=2 \cdot 2$. Three bond wires near the plane of discontinuity ensure equal potential at all ground planes. The CPW centre strip conductor with width $S$ and slot width $W$, are chosen to be compatible with the coaxial launchers of the Wiltron universal test fixture. Thus, the characteristic impedance $Z_{0}$ of the main line is $55 \Omega$.

Experimental results and closed-form equation: The measured return loss and insertion loss of a CPW radial line stub are compared with those of a straight CPW stub in Fig. 2. In this experiment $\theta$ and $R_{2}$ for the radial stub are arbitrarily chosen as $60^{\circ}$ and $5.08 \mathrm{~mm}$, respectively. The length $L$ of the straight stub is $7 \mathrm{~mm}$. The measurements show that for identical $\varepsilon_{\text {r }}$ and rescnance frequency $f_{0}$, the radius $R_{2}$ is $37 \%$ smaller than the length $L$. Further, the radial line stub has a wider bandwidth. The bandwidth of the two structures as a function of the attenuation is compared in Table 1 . The excess loss defined as $1-\left|S_{11}\right|^{2}-\left|S_{21}\right|^{2}$, and determined from the measured $S$ parameters, is small. It is of the order of 0.09 and 0.05 for the radial line stub and straight stub, respectively.

A GCPW radial line stub with $\varepsilon_{r}=2 \cdot 2, D=0.254 \mathrm{~mm}$ and $\theta$ and $R_{2}$ the same as for the CPW radial line stub, has a much wider bandwidth. The characteristics for the GCPW radial line stub are also included in Table 1 . The excess loss is of the order of 0.04 .

Table 1 PERCENTAGE BANDWIDTH OF STRAIGHT AND RADIAL LINE STUB

\begin{tabular}{cccc}
\hline & \multicolumn{3}{c}{ Stub configurations } \\
\cline { 2 - 4 } Attenuation & Straight & CPW radial & GCPW radial \\
\hline dB & $\%$ & $\%$ & $\%$ \\
10 & 18.3 & 25.7 & $58 \cdot 1$ \\
20 & 5.6 & 8.0 & 15.8 \\
30 & 1.6 & 2.5 & 3.5 \\
\hline
\end{tabular}

For a CPW radial line stub, the measured $f_{0}$ as a function of $\theta$ for a fixed $R_{2}$ is shown in Fig. 3. The $f_{0}$ decreases by $20 \%$ as $\theta$ increases from 30 to $90^{\circ}$. It is also worth noting that $R_{1}$ does not remain constant but increases with $\theta$. The excess loss is small and decreases from 0.12 to 0.06 as $\theta$ increases from 30 to $90^{\circ}$.



Fig. 3 Measured resonance frequency of CPW radial line stub against sector angle $S=0.33 \mathrm{~mm}, W=0.234 \mathrm{~mm}, \quad D=0.635 \mathrm{~mm}, \varepsilon_{\mathrm{r}}=10.5, R_{2}=$
$5.08 \mathrm{~mm}$ 


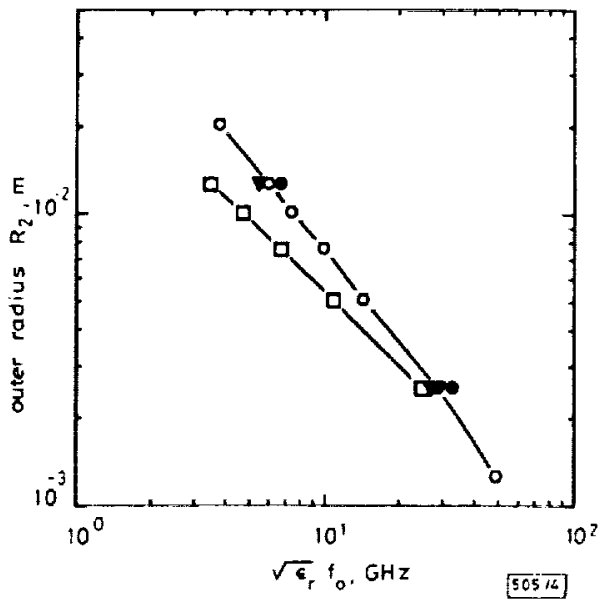

Fig. 4 Measured resonance frequency against outer radius of radial stub for $\theta=60^{\circ}$

(i) $G C P W$ :

(a) $S=0.518 \mathrm{~mm}, W=0.127 \mathrm{~mm}, \quad D=0.254 \mathrm{~mm}, \quad \varepsilon_{r}=2.2$,

$R_{1}=0.3 \mathrm{~mm}$

(ii) $C P W$ :

$O$ (b) $S=0.33 \mathrm{~mm}, W=0.234 \mathrm{~mm}, \quad D=0.635 \mathrm{~mm}, \quad \varepsilon_{r}=10.5$, $R_{1}=0.191 \mathrm{~mm}$

(c) $S=0.33 \mathrm{~mm}, W=0.185 \mathrm{~mm}, \quad D=0.254 \mathrm{~mm}, \varepsilon_{z}=10.5$, $R_{1}=0.191 \mathrm{~mm}$

$\nabla(d) S=0.508 \mathrm{~mm}, W=0.155 \mathrm{~mm}, D=0.635 \mathrm{~mm}, \varepsilon_{r}=6, R_{1}-$ $0.3 \mathrm{~mm}$

$\nabla(e) S=0.152 \mathrm{~mm}, W=0.114 \mathrm{~mm}, D=0.381 \mathrm{~mm}, c_{r}=11 \cdot 7_{t}$ $R_{1}=0.092 \mathrm{~mm}$

Fig. 4 shows the product of $\sqrt{ }\left(\varepsilon_{\mathrm{r}}\right)$ and measured $f_{0}$ as a function of $R_{2}$ for CPW and GCPW radial line stubs. By curve fitting the measured data, the coefficients of a simple closed-form equation to predict $R_{2}$ are determined using a sigma plot. The equations for CPW and GCPW are as follows:

$$
\begin{aligned}
\log \left(R_{2}\right)= & C_{1} \log \left[\sqrt{ }\left(\varepsilon_{r}\right) f_{0}\right] \\
& +C_{2} \log (D)+C_{3} \log \left(R_{1}\right)+C_{4} \\
\log \left(R_{2}\right)= & C_{5} \log \left[\sqrt{ }\left(\varepsilon_{r}\right) f_{0}\right] \\
& +C_{6}\left[\log (D)+\log \left(R_{1}\right)\right]
\end{aligned}
$$

where $f_{0}$ is in gigahertz, $R_{1}, R_{2}$, and $D$ are in metres. The coefficients were determined to be: $C_{1}=-1.0496, C_{2}=$ $0.098375, C_{3}=-0.1401, C_{4}=-1.297, C_{5}=-0.82462$ and $C_{6}=0.21201$, respectively. These equations are valid for the geometry and the range of parameters given in Fig. 4. The range of parameters considered are those typically used in microwave circuit design. The standard error of the equation is at most 0.18 and $0.01 \%$ for the CPW and GCPW, respectively.

Conclusions: A CPW radial line stub resonator is experimentally characterised in terms of its radius, sectoral angle, substrate thickness and relative dielectric constant. A simple closed-form design equation which predicts the resonance radius of the stub for a given set of parameters is also presented. The radial line stub, when compared to a straight stub, has a wider bandwidth and shorter length.

Acknowledgment: The authors are thankful to P. G. Young for fabricating the circuits on silicon substrates.

4th January 1993

R. N. Simons and S. R. Taub (Mail Siop 54-5, NASA Lewis Research Center, 21000 Brookpark Road, Cleveland, Ohio 44!35, USA)

\section{References}

1 KUMAR, M. (Ed.): IEEE 1991 Microwave and Millimeter-Wave Monolithic Circuits Symp. Dig. of Papers (9ICH3016-3)

2 STMONS, R. N, and LII, R. Q.: 'Planar dielectric resonator stabilizod HEMT oscillator integrated with CPW/aperture coupled patch antenna'. IEEE MTT-S Int. Microwave Symp. Dig., 1992, Vol. I, pp. $433-436$

3 ATWATER, H. A.: 'The design of the radial line slub: A useful microstrip circuit element', Microwave J., 1985, 28, (11), pp. 149 -156 